Published in final edited form as:

JAMA Neurol. 2016 March ; 73(3): 358. doi:10.1001/jamaneurol.2015.4185.

\title{
In Reply
}

Joshua W. Miller, PhD, Ralph Green, MD, PhD, and Charles DeCarli, MD

Department of Nutritional Sciences, Rutgers University, New Brunswick, New Jersey (Miller); Department of Medical Pathology and Laboratory Medicine, University of California, Davis (Miller, Green); Department of Neurology, University of California, Davis (DeCarli)

Muratli et al raise the critical issue of whether there is a cause and effect relationship between low vitamin D status and accelerated rates of cognitive decline in older adults. We agree that dementia may lead to reduced vitamin D status owing to reduced exposure to sunlight, decreased dietary intake, and perhaps other circumstances, and therefore, reverse causation cannot be ruled out as an explanation for our results. Indeed, vitamin D status was lower on average in those participants in our study who were diagnosed as having dementia. ${ }^{1}$ However, as we stated in our report, ${ }^{1}$ we do not believe that the significant associations between low vitamin D status and rates of decline in episodic memory and executive function were due to higher prevalence of vitamin D deficiency in participants with dementia because the results remained similar after removing those participants from the statistical analysis. Importantly, participants diagnosed as having mild cognitive impairment and those considered to be cognitively normal had similar baseline vitamin D status.

Muratli et al also question the adequacy of the evaluation of vitamin D status in our study. Specifically, they suggest that measuring parathyroid hormone (PTH) would provide a more accurate diagnosis of vitamin D deficiency in conjunction with measuring serum 25hydroxyvitamin D (25-OHD), the latter being the current clinical standard for assessing vitamin D status. Among its several other functions, PTH promotes the conversion of 25OHD to the active hormone form of vitamin D, 1,25-dihydroxyvitamin D, in the kidney. When vitamin D status falls below a specific threshold, PTH levels in the blood rise and thus may serve as a functional marker of low vitamin D status. Previous studies have suggested that the threshold of vitamin D status (ie, serum 25-OHD) below which PTH starts to rise is different among individuals, and that the consequences of vitamin D deficiency are more likely in those individuals with both low 25-OHD and elevated PTH. ${ }^{2,3}$ Thus, measuring both 25-OHDand PTH may provide a more informative assessment of functional vitamin D status than 25-OHD alone. However, this hypothesis remains to be tested with respect to risk for cognitive decline and dementia in older adults and was beyond the scope of our article.

Muratli et al and Speeckaert and Delanghe raise another potentially relevant issue concerning what is referred to as bio-available vitamin D. Of the circulating 25-OHD in the

Corresponding Author: Joshua W. Miller, PhD, Department of Nutritional Sciences, Rutgers University, 65 Dudley Rd, Room 107, New Brunswick, NJ 08901 (jmiller@ aesop.rutgers.edu).

Conflict of Interest Disclosures: None reported. 
blood, about $90 \%$ is bound to vitamin D-binding proteins, with the remaining $10 \%$ referred to as the free fraction (ie, unbound or bound to albumin). It is hypothesized that only the free fraction is available for direct uptake into tissues. Moreover, the percentage of total serum 25-OHD that is free and bioavailable differs among individuals, most likely owing to polymorphic differences in vitamin D-binding proteins. This is particularly the case in African American individuals, who tend to have lower total serum 25-OHD concentrations than white individuals but similar concentrations of the free fraction. ${ }^{4}$ Thus, measuring only total 25-OHD may not accurately reflect vitamin D status and may limit the predictive usefulness of 25-OHD on outcomes such as cognitive decline and risk for dementia. This hypothesis also remains to be tested.

Despite these limitations, we nonetheless remind that total serum 25-OHD did correlate with rates of cognitive decline in our study and that this association was independent of race/ ethnicity. We reiterate the conclusion of our article ${ }^{1}$ that clinical trials are warranted to determine whether vitamin D supplementation slows cognitive decline and reduces the risk for dementia. ${ }^{1}$ Moreover, these trials should include multiple races and ethnicities and, as discussed here, should include consideration of PTH, free 25-OHD, and genetic polymorphisms in vitamin D-binding proteins.

\section{References}

1. Miller JW, Harvey DJ, Beckett LA, et al. Vitamin D status and rates of cognitive decline in a multiethnic cohort of older adults. JAMA Neurol. 2015; 72(11):1295-1303. [PubMed: 26366714]

2. Kramer CK, Swaminathan B, Hanley AJ, et al. Prospective associations of vitamin D status with $\beta$ cell function, insulin sensitivity, and glycemia: the impact of parathyroid hormone status. Diabetes. 2014; 63(11):3868-3879. [PubMed: 24875346]

3. Sahota O, Mundey MK, San P, Godber IM, Lawson N, Hosking DJ. The relationship between vitamin $\mathrm{D}$ and parathyroid hormone: calcium homeostasis, bone turnover, and bone mineral density in postmenopausal women with established osteoporosis. Bone. 2004; 35(1):312-319. [PubMed: 15207772]

4. Powe CE, Evans MK, Wenger J, et al. Vitamin D-binding protein and vitamin D status of black Americans and white Americans. N Engl J Med. 2013; 369(21):1991-2000. [PubMed: 24256378] 